\title{
Comparison of two transarterial chemoembolization strategies for hepatocellular carcinoma
}

\author{
Akhnur ASSANOVA, Samat ISSAKOV* \\ Central City Clinical Hospital, Kazakhstan
}

Introduction: This retrospective study aimed to compare the efficacy of and tolerance to two center-related conventional transarterial chemoembolization (TACE) strategies in the management of unresectable hepatocellular carcinoma (HCC).

Methods: All HCC patients in whom TACE was initiated in the two centers from July 2008 to June 2016 were included. The TACE strategy performed in center 1 was "on demand" with selective injections of idarubicin, whereas the TACE strategy in center 2 was based "on scheduled" non-selective injections of epirubicin. Toxicity was evaluated using the National Cancer Institute Common Terminology Criteria for Adverse Events.

Results: One hundred and fifty HCC patients were included. Median time to treatment failure was significantly higher in center 1, 13.1 months vs. 7.9 months in center 2 (hazard ratio, 2.32; $p<10-3$ in multivariate analysis). Median overall survival was 21.1 months in center 1 vs. 18.4 months in center $2(p=$ NS). The proportion of grade $\geq 3$ adverse events and mean hospitalisation duration for the overall TACE treatment were significantly greater in center 2 than in center $1: 56 \%$ vs. $32 \%(p<0.01)$ and $14.2 \pm 7.2$ days vs. $10.3 \pm 7.0$ days $(p<0.01)$, respectively.

Conclusions: Our results failed to show any significant survival differences between two center-related TACE strategies but showed a significantly smaller proportion of grade $\geq 3$ adverse events and shorter hospitalisation for the overall treatment when the "on-demand" strategy was used. 